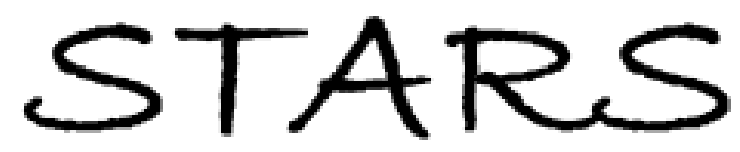

University of Central Florida

STARS

$1-1-1995$

\title{
Signal Gain Due To A Polarization Coupling In An Algaas Channel Wave-Guide
}

\author{
J. S. Aitchison \\ Jin U. Kang \\ University of Central Florida \\ G I. Stege,am \\ University of Central Florida
}

Find similar works at: https://stars.library.ucf.edu/facultybib1990

University of Central Florida Libraries http://library.ucf.edu

This Article is brought to you for free and open access by the Faculty Bibliography at STARS. It has been accepted for inclusion in Faculty Bibliography 1990s by an authorized administrator of STARS. For more information, please contact STARS@ucf.edu.

\section{Recommended Citation}

Aitchison, J. S.; Kang, Jin U.; and Stege,am, G I., "Signal Gain Due To A Polarization Coupling In An Algaas Channel Wave-Guide" (1995). Faculty Bibliography 1990s. 1261.

https://stars.library.ucf.edu/facultybib1990/1261

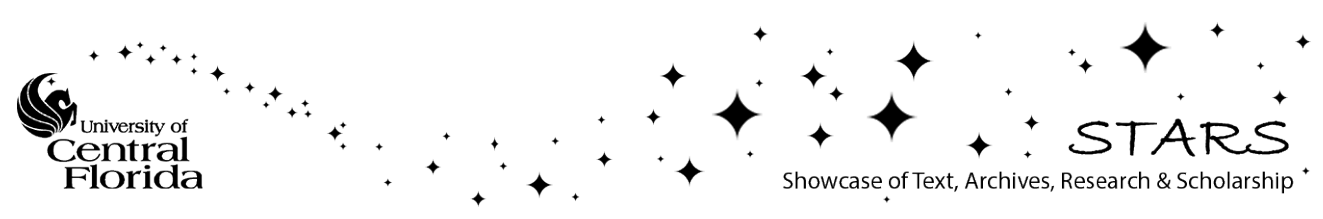




\section{Signal gain due to a polarization coupling in an AlGaAs channel waveguide}

Cite as: Appl. Phys. Lett. 67, 2456 (1995); https://doi.org/10.1063/1.114606

Submitted: 26 July 1995 . Accepted: 21 August 1995 . Published Online: 05 August 1998

J. S. Aitchison, Jin U. Kang, and G. I. Stegeman

\section{ARTICLES YOU MAY BE INTERESTED IN}

The third-order nonlinear optical coefficients of $\mathrm{Si}, \mathrm{Ge}$, and $\mathrm{Si}_{1-x} \mathrm{Ge}_{\mathbf{X}}$ in the midwave and longwave infrared

Journal of Applied Physics 110, 011301 (2011); https://doi.org/10.1063/1.3592270

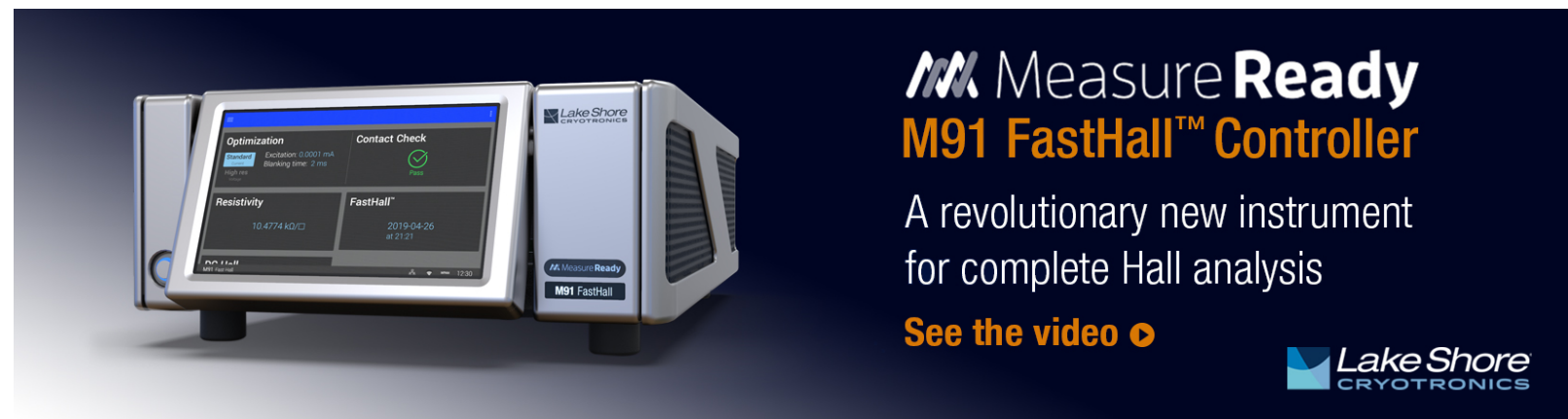




\title{
Signal gain due to a polarization coupling in an AIGaAs channel waveguide
}

\author{
J. S. Aitchison \\ Department of Electronics and Electrical Engineering, Glasgow University, Glasgow G12 8LT, Scotland
}

Jin U. Kang and G. I. Stegeman

CREOL, University of Central Florida, Orlando, Florida 32826

(Received 26 July 1995; accepted for publication 21 August 1995)

\begin{abstract}
We experimentally demonstrate signal gain for picosecond signals due to an orthogonally polarized pump beam in an AlGaAs channel waveguide at a wavelength of $1.55 \mu \mathrm{m}$, below half the band gap. We show as much as $40 \%$ signal gain, or depletion, depending on the polarization states of the pump and the probe. The results are in good agreement with theory. (c) 1995 American Institute of Physics.
\end{abstract}

Interest in all-optical switching has resulted in a great deal of attention being focused on the nonlinearity of semiconductors in the half-band-gap spectral region. The ideal material must have a large nonlinear refraction coefficient, $n_{2}$, small linear, $\alpha_{0}$, and nonlinear, $\alpha_{2}$, loss coefficients, at a wavelength suitable for optical communications. AlGaAs waveguides have proven ideal for such all-optical switching applications due to the ability to tune the half band gap into the $1.55 \mu \mathrm{m}$ low-loss telecommunications window. ${ }^{1}$ However, one of the valid criticisms that has been raised is the lack of gain in this material system that makes fanout impossible. In this letter we demonstrate that polarization coupling can be used to obtain signal gain at the expense of a high intensity pump beam.

Nonlinear coupling between orthogonally polarized beams has been previously studied for application in signal amplification and all-optical switching. Both photorefractive materials $^{2}$ and nonlinear optical fibers ${ }^{3-6}$ have been investigated. However, the slow response time of the photorefractive process makes it unsuitable for operation with short pulses in a high repetition rate system. The ultrafast nonlinear response in weakly birefringent fibers has been used to demonstrate polarization instabilities, all-optical switching, and weak signal controlled switching. ${ }^{4-6}$ However, the low nonlinearity of silica glass means that fibers of $1-10 \mathrm{~m}$ are required. Although it is possible to have polarization coupling between two orthogonally polarized beams in millimeter long channel waveguide due to the ultrafast Kerr nonlinearity, the material requirements, i.e., large nonlinearity and low absorptive losses are not easy to satisfy. To date, polarization switching that involves the equal excitation of the two eigenmodes has been demonstrated in AlGaAs channel waveguides below half the band gap. ${ }^{7}$ In this letter, we experimentally show strong energy coupling between two orthogonally polarized beams resulting in weak signal gain in an AlGaAs channel waveguide using the off-resonant boundelectronic Kerr nonlinearity at $1.55 \mu \mathrm{m}$. This intensity dependent coupling between two orthogonally polarized beams is demonstrated in a typical pump/probe geometry and the experimental results show good agreement with theory.

The polarization coupling occurs due to the nonlinear polarization grating induced by two orthogonally polarized beams. In order to obtain efficient coupling between two orthogonally polarized beams, two conditions must be satis- fied, namely, the difference between the propagation constants must be less than or of order of the nonlinear (intensity-dependent) coupling constant and a sample length must be of order of the beat length, or longer. In the case of photorefractive materials, the polarization coupling has been demonstrated in cubic crystals such as GaAs, InP, and CdTe where the material birefringence is zero. ${ }^{2} \mathrm{AlGaAs}$ is also cubic and therefore has no linear birefringence. However, when used in waveguide structures, an induced waveguide birefringence occurs. The waveguide birefringence can be controlled by properly designing the waveguides and hence it can be made small. Although the waveguide used in this experiment is not optimized to minimize the birefringence, nevertheless the birefringence was small enough to demonstrate the desired effects. We estimated the difference between the mode indices of TE (transverse electric) and TM (transverse magnetic) modes to be about 0.0003 , giving a beat length of $5 \mathrm{~mm}$.

The experiment was performed with a $\mathrm{NaCl}$ color center laser (CCL) operating at a wavelength of $1.55 \mu \mathrm{m}$ and producing $5 \mathrm{ps}$ pulses at a repetition rate of $76 \mathrm{MHz}$. Using picosecond, rather than subpicosecond, pulses avoids the problems of crosstalk between orthogonally polarized signals, due to the Raman effect. ${ }^{8}$ A $1.74 \mathrm{~cm}$ long, strip-loaded AlGaAs channel waveguide was used for this experiment. The high index, guiding region is made of $\mathrm{Al}_{0.18} \mathrm{Ga}_{0.82} \mathrm{As}$ and the upper and lower cladding regions of $\mathrm{Al}_{0.24} \mathrm{Ga}_{0.76} \mathrm{As}$. Waveguides were formed using standard optical lithography and reactive ion etching.

The governing equations for polarization coupling between two orthogonally polarized beams are shown below:

$$
\begin{aligned}
\frac{d E_{e}}{d z}= & -\frac{\alpha}{2}+i k_{0} n_{2, e}\left[\left|E_{e}\right|^{2} E_{e}+\Delta\left|E_{m}\right|^{2} E_{e}\right. \\
& \left.+\frac{\Delta}{2} E_{m}^{2} E_{e}^{*} \exp (-2 i \Delta k z)\right], \\
\frac{d E_{m}}{d z}= & -\frac{\alpha}{2}+i k_{0} n_{2, m}\left[\left|E_{m}\right|^{2} E_{m}+\Delta\left|E_{e}\right|^{2} E_{m}\right. \\
& \left.+\frac{\Delta}{2} E_{e}^{2} E_{m}^{*} \exp (2 i \Delta k z)\right],
\end{aligned}
$$


Delay line

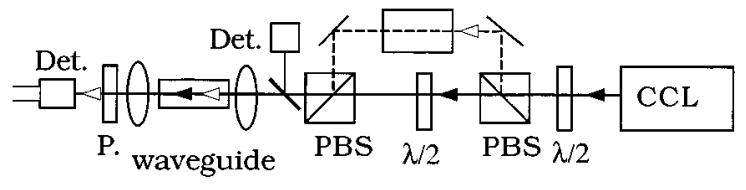

FIG. 1. Schematic diagram of experimental setup showing the color center laser (CCL), half-wave plates $(\lambda / 2)$, polarizing beam splitters (PBS), output polarizer $(\mathrm{P})$, and the detectors (Det).

where $E_{e}$ and $E_{m}$ are the electric fields for the TE and TM modes, respectively, $\alpha$ is the linear loss coefficient, which is about $0.1 \mathrm{~cm}^{-1}, k_{0}$ is the propagation constant in the vacuum, $n_{2, e}$ and $n_{2, m}$ are the nonlinear refractive index coefficient for the TE and TM fields, respectively, $\Delta$ is the ratio between the cross and self phase modulations, and $\Delta k$ is the wave-vector mismatch defined as $k_{0}\left(n_{e}-n_{m}\right)$, where $n_{e}$ and $n_{m}$ are the effective indices for TE and TM fields, respectively. The last term in the equation, which is typically negligible in most linear waveguide problems due to the large wave-vector mismatch, provides the energy coupling between the orthogonally polarized fields.

Experiments were performed using the pump-probe geometry shown in Fig. 1. The pump and the signal beam were generated using a half-waveplate and polarizing beam splitter (PBS); the two beams were then recombined using a second PBS and end-fire coupled into the channel waveguide using a microscope objective. At the output a second microscope objective was used to collect the light and a polarizer was used to block the pump beam. Using a TM pump, we observed an increase in the TE-polarized output signal due to polarization coupling. This is shown in Fig. 2, where the output signal intensity is plotted as a function of pump intensity. Note that as pump power is increased, the signal output increases and shows gain of as much as $40 \%$. However, when the polarization of the pump and the signal was reversed, depletion of the signal beam was observed. This is shown in Fig. 3.

The theoretical curves based on the reported values are

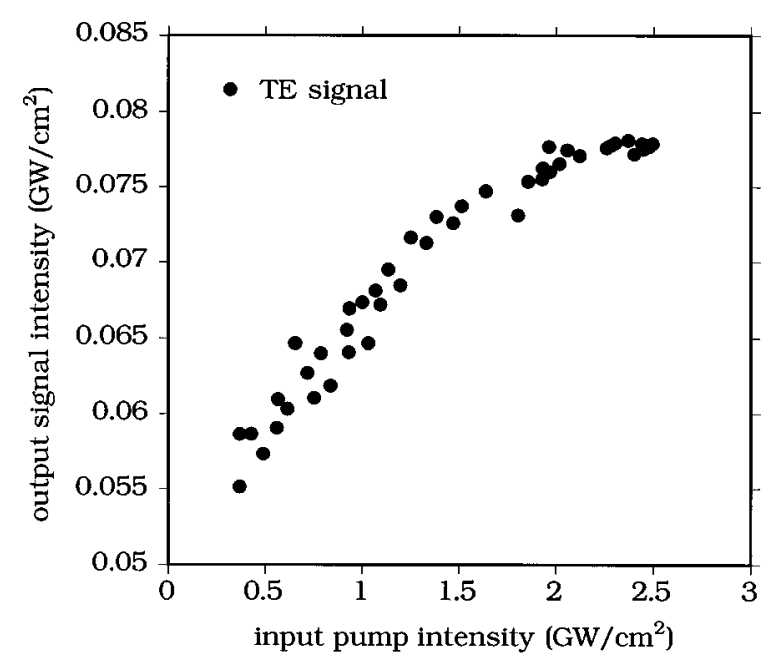

FIG. 2. TE signal output intensity as a function of TM pump input intensity.

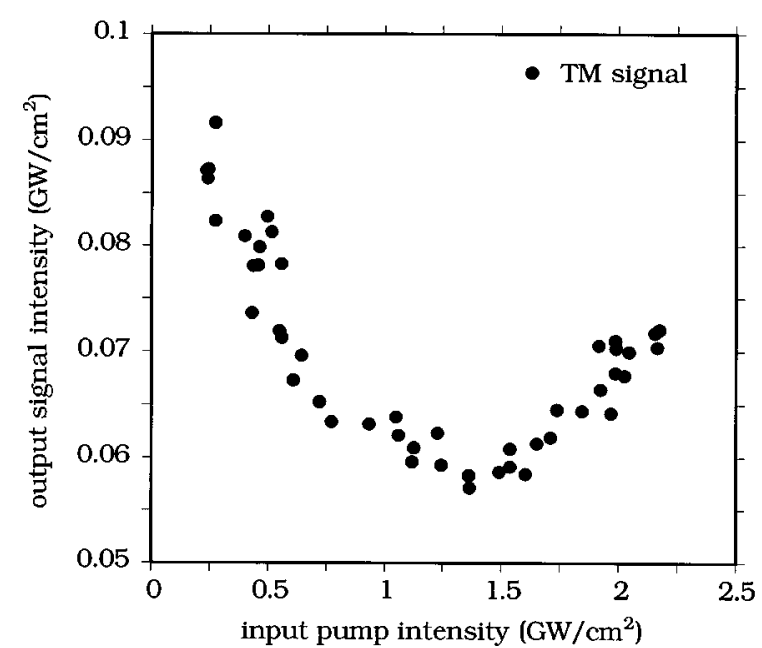

FIG. 3. TM signal output intensity as a function of TE pump input intensity.

shown in Fig. 4 for both the gain and depletion cases. ${ }^{9}$ The parameters based on the reported values were varied within the experimental uncertainty. We used values of $n_{2, e}$ and $n_{2, m}$ of $1.5 \times 10^{-13} \mathrm{~cm}^{2} / \mathrm{W}$ and $1.3 \times 10^{-13} \mathrm{~cm}^{2} / \mathrm{W}\left(n_{e}\right.$ $-n_{m}$ ) of 0.000314 , and $\Delta$ of 0.92 . The solid and dotted lines represent the TE signal with a TM pump and the TM signal with a TE pump, respectively. The predicted gain for a TEpolarized signal and depletion for a TM-polarized signal is consistent with the experimental results.

The case considered above occurs when the initial relative phase difference between the two beams is zero. When the phase difference is $\pi / 2$, the process is reversed so that gain occurs for a TM signal with a TE pump, and depletion for a TE signal with a TM pump. This was verified experimentally.

In conclusion, we demonstrated ultrafast signal gain due to polarization coupling in $\mathrm{AlGaAs}$ channel waveguides for $1.55 \mu \mathrm{m}$ picosecond pulses. For a waveguide with a smaller wave-vector mismatch, a larger gain is expected. Such a de-

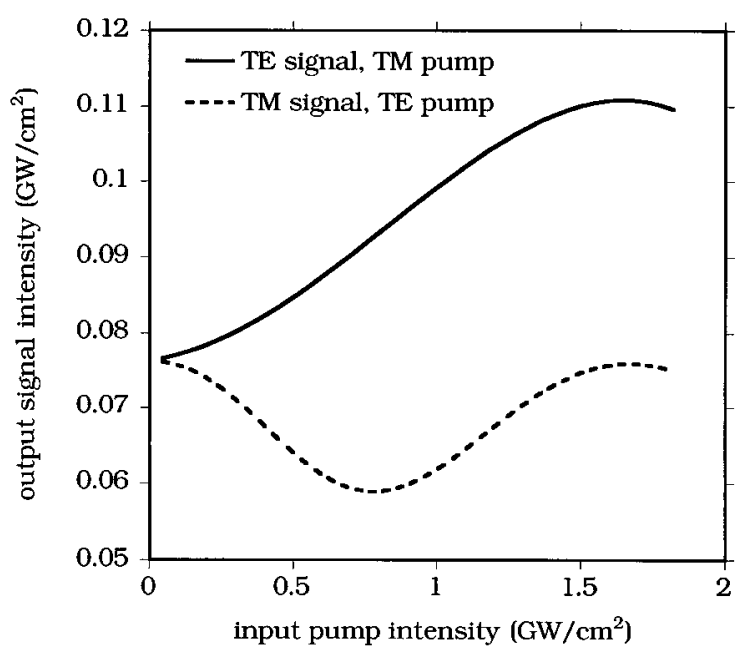

FIG. 4. Theoretical curves showing both gain and depletion of signal as a function of pump input intensity. Solid and dotted lines represent TE signal for TM pump and TM signal for TE pump, respectively. 
vice can play an important role in AlGaAs applications because it can be used in combination with a switching device to implement fanout.

The work in the UK was supported by the SERC through the nonlinear optics initiative, and at CREOL was sponsored by NSF.

${ }^{1}$ Reviewed in G. I. Stegeman and A. Miller, Photonic Switching, edited by J. Midwinter (Academic, Orlando, 1992), Vol. I, p. 81.

${ }^{2}$ For example, J. C. Fabre, J. M. C. Jonathan, and G. Roosen, J. Opt. Soc. Am. B 5, 1730 (1988).

${ }^{3}$ H. G. Winful, Opt. Lett. 11, 33 (1986).
${ }^{4}$ S. Trillo, S. Wabnitz, R. H. Stolen, G. Assanto, C. T. Seaton, and G. I. Stegeman, Appl. Phys. Lett. 49, 1224 (1986).

${ }^{5}$ S. Trillo, S. Wabnitz, W. C. Banyai, N. Finlayson, C. T. Seaton, G. I. Stegeman, and R. H. Stolen, IEEE J. Quantum Electron. 25, 104 (1988).

${ }^{6}$ C. G. Krautschik, G. I. Stegeman, and R. H. Stolen, Appl. Phys. Lett. 61, 1751 (1992).

${ }^{7}$ C. C. Yang, A. Villeneuve, G. I. Stegeman, C.-H. Lin, and H.-H. Lin, Opt. Lett. 18, 1487 (1993).

${ }^{8}$ Y. H. Kao, M. N. Islam, J. M. Saylor, R. E. Slusher, and W. S. Hobson, "Raman effect in AlGaAs waveguides for all-optical applications," Digest of QELS'95 (Optical Society of America, Washington, DC, 1995), pp. $115-116$.

${ }^{9}$ D. C. Hutchings, J. S. Aitchison, B. S. Wherrett, G. T. Kennedy, and W. Sibbett, Opt. Lett. 20, 991 (1995). 